\title{
Contextuality and bundle diagrams
}

\author{
Kerstin Beer and Tobias J. Osborne \\ Institut für Theoretische Physik, Leibniz Universität Hannover, 30167 Hannover, Germany
}

(Received 3 August 2018; published 20 November 2018)

\begin{abstract}
Contextuality describes the local consistency but global inconsistency of data. In this paper, we review and generalize the bundle diagram representation introduced in [S. Abramsky, R. S. Barbosa, K. Kishida, R. Lal, and S. Mansfield, Contextuality, Cohomology and Paradox, 24th EACSL Annual Conference on Computer Science Logic, Vol. 41 (CSL, 2015), p. 211-228 ] to graphically demonstrate the contextuality of diverse empirical models (a collection of probability distributions for jointly measurable observables).
\end{abstract}

DOI: 10.1103/PhysRevA.98.052124

\section{INTRODUCTION}

Contextuality - the general impossibility of assigning predetermined outcomes to measurements of quantum observables in a noncontextual way-is a crucial feature of quantum mechanics [1,2]. Quantum contextuality has a wide variety of applications; for example, understanding Bell nonlocality [3-9]. Recently, it has been shown that in specific models of quantum computation, contextuality is a necessary resource [10-13]. There has been rapid recent progress in understanding contextuality, both theoretically [10,14-18] and experimentally [19-28], leading to many insights into the behavior of contextual models. However, many mysteries remain and a general theory of contextuality analogous to the theory of quantum entanglement, remains to be achieved [29-31].

Several frameworks have been developed to study quantum contextuality exploiting, variously, graph and hypergraph theory, sheaf theory, and cohomology theory [14-16,32,33]. Most relevant to this work are a sequence of works by Abramsky and coworkers aiming to quantify contextuality using the technology of sheaf theory. Here one conceives of an empirical model - a collection of probability distributions for jointly measurable observables — as a kind of "vector bundle": the observables of the empirical model are points in the "base space" where two points are connected if they may be jointly measured (i.e., if they are in the same context). The possible measurement outcomes are then associated to the contexts (the edges) similar to transition maps. This analogy is not just superficial as one can exploit the theory of sheaves, developed to generalize that of vector bundles, to exactly dure the data of an empirical model. Exploiting results from sheaf theory, one can formulate various obstructions to noncontextuality in terms of properties of the sections of the sheaf.

The formalism of sheaf theory is very powerful and many key results have been obtained via its application. However, it is challenging for the newcomer to appreciate its utility. In particular, although it is motivated by vector bundle theory, a geometrical interpretation of the sheaf-theoretic formulation of an empirical model may be unclear. To clarify its geometrical aspects Abramsky et al. [34] introduced a graphical method via bundle diagrams to visualize empirical models, particularly in the setting where there are two agents who can each choose to measure one of two possible observables. Bundle diagrams are visually striking and of considerable utility in understanding contextuality. For this reason we were interested in generalizing the bundle diagram approach as a pedagogical tool to study empirical models involving more agents and measurement settings.

In this paper, we study a variety of empirical models going beyond the two-agent scenario and explain how to adapt the bundle diagram technology of Abramsky et al. [34] and Carù [35] to illustrate the contextuality of these empirical models. We discuss strong contextuality-where the possible local measurement outcomes cannot all be preassigned-in detail here. Raussendorf [36] has shown that any (classical and quantum) measurement-based computation with $\mathbb{Z}_{2}$-linear classical processing computing a nonlinear boolean function constitutes a proof of strong contextuality. If a noncontextual hidden variable model, where the outcomes associated with measurements are predetermined by (local) value assignments, describe a quantum measurement-based computation with $\mathbb{Z}_{2}$-linear classical processing, it is restricted to computing linear functions.

In the first section, we review the sheaf-theoretic formulation of empirical models and therefore recall the most important definitions from Ref. [18]. Introducing the bundle diagram representation helps one to geometrically understand the sheaf structure of the empirical models. Furthermore, we review how one can deduce contextuality from the nonexistence of a global section. In the following, we represent a measurement-based quantum computational model via a bundle diagram. We then graphically illustrate that, for a Greenberger-Horne-Zeilinger type scenario, there exists no empirical model with a global section. We conclude with a discussion of examples arising from the cluster state on a ring.

\section{THE SHEAF-THEORETIC STRUCTURE OF EMPIRICAL MODELS}

The aim of this section is to understand how to associate a sheaf to an empirical model and to describe contextuality via this mathematical structure. We restrict ourselves to the case where Pauli measurement operations are the observables. 
TABLE I. Empirical model for $|\Psi\rangle$.

\begin{tabular}{cccccc}
\hline \hline$A$ & $B$ & 00 & 10 & 01 & 11 \\
\hline$X_{A}$ & $X_{B}$ & $1 / 2$ & 0 & 0 & $1 / 2$ \\
$X_{A}$ & $Z_{B}$ & $1 / 4$ & $1 / 4$ & $1 / 4$ & $1 / 4$ \\
$Z_{A}$ & $X_{B}$ & $1 / 4$ & $1 / 4$ & $1 / 4$ & $1 / 4$ \\
$Z_{A}$ & $Z_{B}$ & $1 / 2$ & 0 & 0 & $1 / 2$ \\
\hline \hline
\end{tabular}

Throughout this section, we very closely follow the papers of Abramsky and coworkers [17,18,34,35].

In the basic setting there are several agents, who can each select from a set of measurements and observe outcomes. We call the procedure whereby each agent performs a measurement on their system and observes an outcome, an event. A probability distribution on these events results from repeated trials.

Definition 1: An empirical model is a family of probability distributions on events, one for each choice of measurements. A set of allowed jointly measurable observables is called a measurement context.

We exemplify the theory in terms of a bipartite qubit model, where each of the two parties, Alice and Bob, can apply a Pauli measurement operation to the state

$$
\begin{aligned}
|\Psi\rangle & =\frac{1}{\sqrt{2}}\left(|0\rangle_{A} \otimes|0\rangle_{B}+|1\rangle_{A} \otimes|1\rangle_{B}\right) \\
& \equiv \frac{1}{\sqrt{2}}(|00\rangle+|11\rangle) .
\end{aligned}
$$

We choose for the allowed observables $X=|0\rangle\langle 1|+| 1\rangle\langle 0|$ and $Z=|0\rangle\langle 0|-| 1\rangle\langle 1|$ and index the measurement operations according to whether Alice or Bob carries out the measurement. The measurement setting where Alice measures $X_{A}=X \otimes \mathbb{1}$ and Bob $X_{B}=\mathbb{1} \otimes X$ is an example of a pair of jointly measurable observables (the two observables commute). For this bipartite system, we take for our contexts the following four sets of jointly measurable observables:

$$
\begin{gathered}
C_{1}=\left\{X_{A}, X_{B}\right\}, \\
C_{2}=\left\{X_{A}, Z_{B}\right\}, \\
C_{3}=\left\{Z_{A}, X_{B}\right\}, \quad \text { and } \\
C_{4}=\left\{Z_{A}, Z_{B}\right\} .
\end{gathered}
$$

The outcomes of the measurements can be described by the eigenvalues, which are \pm 1 for both $X$ and $Z$. To this end, we label the outcome $(-1)^{j}$ where $j \in\{0,1\}$ is a bit indicating the outcome. Thus, the probabilities that the measurement outcome is $j$ for Alice and $k$ for Bob may be summarized in Table I.

\section{A. Joint measurability structures and abstract simplicial complexes}

Abramsky et al. [34] introduced a diagrammatic representation-further developed in Ref. [35] - to depict empirical models whereby measurements are represented as vertices in a "base space" and possible outcomes as "fibers" above the base.

To describe this representation, we first note [37,38] that a contextual model, or joint measurability structure, may be represented via a combinatorial object known as an abstract simplicial complex. Recall that a Positive Operator Valued Probability Measure (POVM) is a map $A: \Omega \rightarrow \mathcal{B}^{+}(\mathcal{H})$ from an outcome set (which we take to be finite from now on) to the convex cone of positive operators on a Hilbert space $\mathcal{H}$ such that

$$
\sum_{j \in \Omega} A(j)=\mathbb{I} .
$$

We say that a POVM $A$ with outcome set $\Omega_{1} \times \cdots \times \Omega_{n}$ marginalizes to a set of POVMs $\left\{A_{1}, \ldots, A_{n}\right\}$ with outcome sets $\left\{\Omega_{1}, \ldots, \Omega_{n}\right\}$, respectively, if

$$
\sum_{j_{1}, \ldots, \widehat{j}_{k}, \ldots, j_{n}} A\left(j_{1}, \ldots, j_{n}\right)=A_{k}\left(j_{k}\right)
$$

for all $k=1,2, \ldots, n$, where the hat means that the variable is excluded from the summation. If, for a given set $\left\{A_{1}, \ldots, A_{n}\right\}$ of POVMs there is such a measurement $A$ marginalizing to $\left\{A_{1}, \ldots, A_{n}\right\}$, then we say they are jointly measurable. Thus, if a set of POVMs is jointly measurable then so is any subset of them.

An elegant combinatorial object which naturally dures the structure of a contextual model is that of an abstract simplicial complex.

Definition 2: A family $\Delta$ of nonempty subsets of a set $M$ is an abstract simplicial complex if, for every set $U \in \Delta$ and every nonempty subset $V \subset U, V$ also belongs to $\Delta$. The finite sets belonging to $\Delta$ are called faces and the vertices are the elements of the set $\cup \Delta$. (We henceforth assume that $M=\bigcup \Delta$.

An abstract simplicial complex $\Delta$ gives rise to a topological space by endowing it with the Alexandroff topology by defining a subset $\mathcal{U} \subset \Delta$ to be closed if and only if $\mathcal{U}$ is itself an abstract simplicial complex, i.e., $\forall U \in \mathcal{U}$ if $V \subset U$ then $V \in \mathcal{U}$. The open sets are hence generated by stars, where for a face $\sigma \in \Delta$ we set $\operatorname{star}(\sigma) \equiv\{\tau \in \Delta \mid \sigma \in \tau\}$. In particular, this means that upper sets in the poset of faces with respect to inclusion, i.e., maximal faces, are open.

We henceforth take for the vertices of the abstract simplicial complex associated to a contextual model the set of all allowed observables: a set of vertices form a face whenever the corresponding measurements can be performed jointly. Thus contexts correspond to faces of a such complex. This complex is called the base.

Continuing the two-qubit example above, the base suitable to the above-described model is the complex $\left\{\left\{X_{A}\right\},\left\{X_{B}\right\},\left\{Z_{A}\right\},\left\{Z_{B}\right\}, C_{1}, C_{2}, C_{3}, C_{4}\right\}$. This may be recognized as a square depicted in Fig. 1, where the four vertices represent the observables and the edges the contexts.

\section{B. Bundle diagrams}

We can enhance the abstract simplicial complex representation by including information about the possible measurement outcomes of the observables of a context. To this end, we attach, above each vertex, the fiber (or, more correctly, 


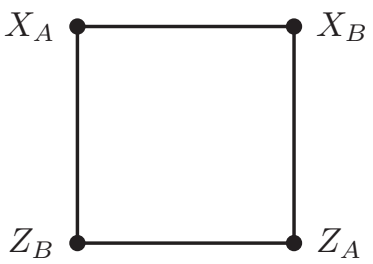

FIG. 1. Base of the bundle diagram.

the stalk) of possible measurement outcomes, which in this example are either 0 or 1 . This can be seen in Fig. 2 .

Depending on the empirical model, not all measurement outcomes can occur. We illustrate this by connecting elements of the fibers above a face (i.e., above a context) if the corresponding outcome combination has a probability strictly larger than 0 . For the two-qubit example, we depict this by connecting possible outcomes with purple lines.

The example illustrated in Fig. 3 is noncontextual. Before proceeding, it is instructive to give an example of what a contextual empirical model looks like. To this end, we consider the Popescu-Rohrlich box [39], which corresponds to a joint measurement scenario of a particular extremal state of a generalized probabilistic theory whose correlations exceed those allowed by quantum mechanics. Measurements of this state give rise to the empirical model (Table II). The corresponding bundle diagram is illustrated in Fig. 4. This model is contextual.

At this point, it is clear that the bundle diagram representation can only describe a possibilistic model as it only contains information about what possible outcomes can occur, and not the corresponding probability. Nevertheless, we will see that such bundle diagrams are of great utility for representing the contextuality of an empirical model.

To more precisely describe a useful graphical representation for an empirical model, it is helpful to first introduce some of the mathematical terminology of sheaf theory.

\section{Sheaf structure}

We denote by $M$ the vertices of an abstract simplicial complex $\Delta$ corresponding to a contextual model. It is assumed throughout that the set of allowed observables $M$ for the model and the set of outcomes $O$ are finite. We give $M$ the

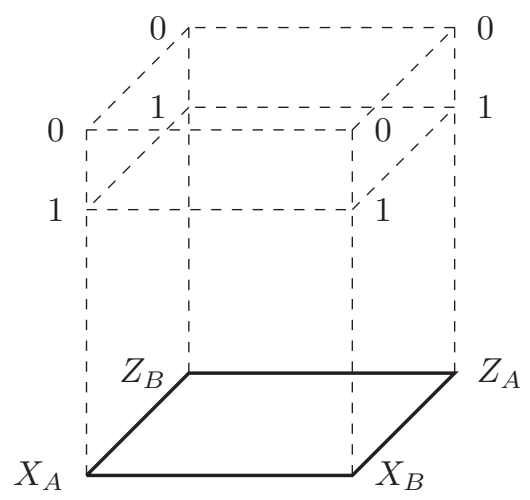

FIG. 2. Fibers of the bundle diagram.

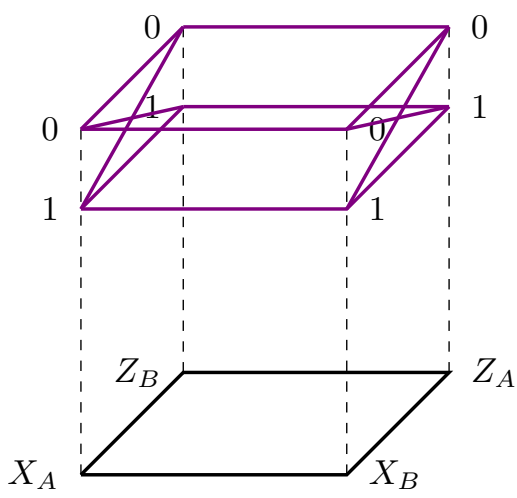

FIG. 3. Bundle diagram for the empirical model of Table I.

discrete topology by defining any subset of $M$ to be open, i.e., the topology for $M$ is the power set $\mathcal{P}(M)$. (Note: this topological space is very different from the natural topological space $\Delta$ given by the Alexandroff topology.) We first build a structure called a presheaf of sets over the space $(M, \mathcal{P}(M))$. This presheaf is intended to dure all possible measurement outcomes, regardless of whether the observables are jointly measurable or not (note that, depending on whether $U$ is a context, there may or may not be a joint measurement for the observables in $U$ ). We do this by associating to every subset $U \subset M$ the set of all functions from $U$ to $O$. This is a finite set, denoted $O^{U}$, with cardinality $|O|^{|U|}$. We call a function $s: U \rightarrow O$ a section over $U$. A section in $\mathcal{E}(M)$ is called a global section.

Definition 3: If $s: U^{\prime} \rightarrow O$ is a function and $U \subseteq U^{\prime}$, we write $\left.s\right|_{U}: U \rightarrow O$ for the restriction of $s$ to $U$. We define the restriction map

$$
\operatorname{res}_{U}^{U^{\prime}}: \mathcal{E}\left(U^{\prime}\right) \rightarrow \mathcal{E}(U)
$$

via

$$
\left.\operatorname{res}_{U}^{U^{\prime}}(s) \equiv s\right|_{U}
$$

The map $\operatorname{res}_{U}^{U^{\prime}}$ endows $\mathcal{E}$ with the structure of a presheaf as it enjoys the additional properties that $\operatorname{res}_{U}^{U^{\prime}} \circ \operatorname{res}_{U^{\prime}}^{U^{\prime \prime}}=\operatorname{res}_{U}^{U^{\prime \prime}}$ for $U \subset U^{\prime} \subset U^{\prime \prime}$ and $\operatorname{res}_{U}^{U}=\mathrm{id}_{U}$.

This presheaf $\mathcal{E}$ of events has two important additional properties.

Definition 4: (1) Locality: if $\left\{V_{j}\right\}$ is a covering of $U$ and if $s, t \in \mathcal{E}(U)$ are elements such that $\left.s\right|_{V_{j}}=\left.t\right|_{V_{j}}$ for all $V_{j}$, then $s=t$.

(2) Gluing: suppose $\left\{V_{j}\right\}$ is an open covering of $U \subset$ $M$ and we have a family of sections $\left\{s_{j} \in \mathcal{E}\left(V_{j}\right)\right\}$ with the property that for all $j, k$,

$$
\left.s_{j}\right|_{V_{j} \cap V_{k}}=\left.s_{k}\right|_{V_{j} \cap V_{k}},
$$

TABLE II. Empirical model for the Popescu-Rohlich box.

\begin{tabular}{lccccl}
\hline \hline$A$ & $B$ & 00 & 10 & 01 & 11 \\
\hline$N_{A}$ & $N_{B}$ & $1 / 2$ & 0 & 0 & $1 / 2$ \\
$N_{A}$ & $M_{B}$ & $1 / 2$ & 0 & 0 & $1 / 2$ \\
$M_{A}$ & $N_{B}$ & $1 / 2$ & 0 & 0 & $1 / 2$ \\
$M_{A}$ & $M_{B}$ & 0 & $1 / 2$ & $1 / 2$ & 0 \\
\hline \hline
\end{tabular}




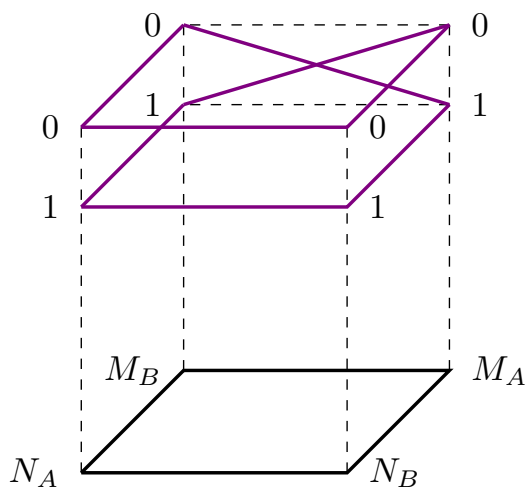

FIG. 4. Bundle diagram for the empirical model of Table II.

then there is a unique section $s \in \mathcal{E}(U)$ such that $\left.s\right|_{V_{j}}=s_{j}$ for all $j$.

Any presheaf obeying locality and gluing is called a sheaf, which, in this case, we refer to as the sheaf of events. The sheaf condition says that we can uniquely glue together compatible local data, where "compatible" means that measurement outcomes agree on observables common to contexts.

Let $U \in \Delta$ be a context: we now exclude events with probabilities strictly equal to 0 and write $\mathcal{F}(U)$ for the set of sections over the context. For example, each of the purple lines directly above the set of measurements $C_{3}$ in Fig. 3 corresponds to a section in the set $\mathcal{E}\left(C_{3}\right)$ :

$$
\begin{aligned}
\mathcal{E}\left(C_{3}\right)=\left\{s_{C_{3} 00}\left(Z_{A}\right)\right. & =0, s_{C_{3} 00}\left(X_{B}\right)=0, \\
s_{C_{3} 01}\left(Z_{A}\right) & =0, s_{C_{3} 01}\left(X_{B}\right)=1, \\
s_{C_{3} 10}\left(Z_{A}\right) & =1, s_{C_{3} 10}\left(X_{B}\right)=0, \\
s_{C_{3} 11}\left(Z_{A}\right) & \left.=1, s_{C_{3} 11}\left(X_{B}\right)=1\right\} .
\end{aligned}
$$

By only including sections with a nonzero probability of occurring, we can obtain a subpresheaf $\mathcal{F}$ of $\mathcal{E}$. This is done by associating to sets $U \subset M$ built from the unions of two or more contexts only those sections which are compatible on the overlaps between the constituent contexts. (Abramsky and coworkers [18] describe some additional properties of this subpresheaf; however, we do not need them for this discussion.)

A key role is played throughout this subject by the existence of global sections [18] because a global section glues together a compatible family on a presheaf and allocates a predetermined outcome to each measurement. In this case, the measurement outcomes depend only on the measurement operator and not the context. If every section $s$ belongs to a compatible family, then the model is called noncontextual. Such a model is described by a hidden-variable model. If there is a section which does not belong to a compatible family, then the model is said to be logically contextual. If no section belongs to a compatible family, then the model is strongly contextual.

Abramsky and his coworkers [18] determined locality and noncontextuality in terms of the existence of global sections:

Proposition 5: The existence of a global section for an empirical model implies the existence of a noncontextual deterministic hidden-variable model which realizes it.

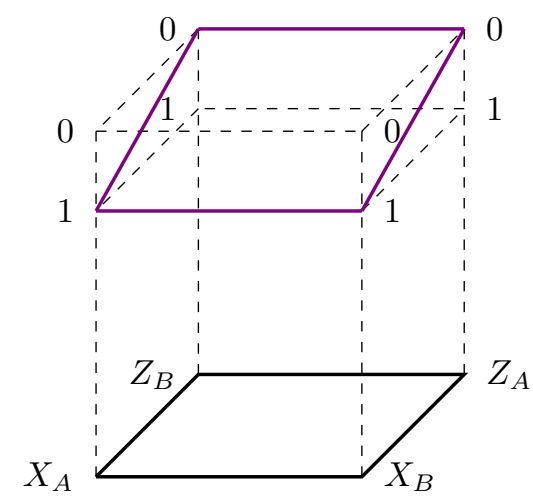

FIG. 5. Example for a global section.

Thus, given an empirical model, we have to check whether we can extend every section to a global one, i.e., we can realize every possible outcome combination for a context in a local hidden variable model. If this is the case, the model is noncontextual.

A global section in the bundle diagram is represented by a closed path traversing all the fibers exactly once. This observation allows us to graphically determine the contextuality of an empirical model. The above-discussed model is noncontextual, because every section is extendable to a global one. An example of a global section is depicted in Fig. 5.

\section{CONTEXTUALITY AS A RESOURCE FOR (QUANTUM) COMPUTATION}

In a key paper, Anders and Browne [10] argued that in MBQC a parity computer achieves classical universality using contextuality. This argument exploits joint measurements of a Greenberger-Horne-Zeilinger-type state $|\Psi\rangle=\frac{1}{\sqrt{2}}(|001\rangle-$ $|110\rangle)$ as a resource. By carrying out measurements of this state, a classical parity computer only able to implement NOT $=|1\rangle\langle 0|+| 0\rangle\langle 1|$ and CNOT $=|1\rangle\langle 1|\otimes X+| 0\rangle\langle 0| \otimes \mathbb{1}$ operations may be augmented to carry out any deterministic computation. The canonical gate enabled by measurements of the GHZ state is the NAND-gate in Table III.

By performing three measurements on the GHZ state, it is possible to deterministically implement this gate [40]. Our objective for this section is to explain how to represent the resulting contextual model via a generalized bundle diagram and explore its contextuality in terms of the nonexistence of global sections.

We build a joint measurability structure for the AndersBrowne example as follows. We take for the set of observables $M=\left\{X_{A}, X_{B}, X_{C}, Y_{A}, Y_{B}, Y_{C}\right\}$ with $Y=-i|0\rangle\langle 1|+$

TABLE III. Truth table for NAND-gate.

\begin{tabular}{ccc}
\hline \hline $\mathrm{a}$ & $\mathrm{b}$ & NAND \\
\hline 0 & 0 & 1 \\
0 & 1 & 1 \\
1 & 0 & 1 \\
1 & 1 & 0 \\
\hline \hline
\end{tabular}


TABLE IV. Empirical model for the Anders-Browne example.

\begin{tabular}{cccccccccccc}
\hline \hline & $A$ & $B$ & $C$ & 000 & 001 & 010 & 011 & 100 & 101 & 110 & 111 \\
\hline$C_{1}$ & $X_{A}$ & $X_{B}$ & $X_{C}$ & 0 & $1 / 4$ & $1 / 4$ & 0 & $1 / 4$ & 0 & 0 & $1 / 4$ \\
$C_{2}$ & $X_{A}$ & $Y_{B}$ & $Y_{C}$ & 0 & $1 / 4$ & $1 / 4$ & 0 & $1 / 4$ & 0 & 0 & $1 / 4$ \\
$C_{3}$ & $Y_{A}$ & $X_{B}$ & $Y_{C}$ & 0 & $1 / 4$ & $1 / 4$ & 0 & $1 / 4$ & 0 & 0 & $1 / 4$ \\
$C_{4}$ & $Y_{A}$ & $Y_{B}$ & $X_{C}$ & $1 / 4$ & 0 & 0 & $1 / 4$ & 0 & $1 / 4$ & $1 / 4$ & 0 \\
\hline \hline
\end{tabular}

$i|1\rangle\langle 0|$. The choice of which measurements Alice and Bob perform depend on the inputs $a$ and $b$ of the NAND-gate they wish to compute. Charlie measures his observable according to the value of a third supplemented input $a \oplus b$. If the input is 0 , an $X$ measurement is carried out and for 1 a $Y$ measurement is made. We can summarize this procedure in terms of the following four contexts:

$$
\begin{gathered}
C_{1}=\left\{X_{A}, X_{B}, X_{C}\right\}, \\
C_{2}=\left\{X_{A}, Y_{B}, Y_{C}\right\}, \\
C_{3}=\left\{Y_{A}, X_{B}, Y_{C}\right\}, \quad \text { and } \\
C_{4}=\left\{Y_{A}, Y_{B}, X_{C}\right\} .
\end{gathered}
$$

We use the basis

$$
|+\rangle=\frac{1}{\sqrt{2}}(|0\rangle+|1\rangle), \text { and }|-\rangle=\frac{1}{\sqrt{2}}(|0\rangle-|1\rangle)
$$

for the $X$ measurements and

$$
|\circlearrowleft\rangle=\frac{1}{\sqrt{2}}(|0\rangle+i|1\rangle) \text {, and }|\circlearrowright\rangle=\frac{1}{\sqrt{2}}(|0\rangle-i|1\rangle),
$$

for the $Y$ measurements. Measuring these contexts leads to the empirical model in Table IV.

The abstract simplicial complex $\Delta$ corresponding to this example is depicted in Fig. 6.

We obtain a generalized bundle diagram for the AndersBrowne example by depicting the possible outcomes of a joint measurement of a context not as a line, but instead as a triangular facet in Fig. 7. We are lucky that in three

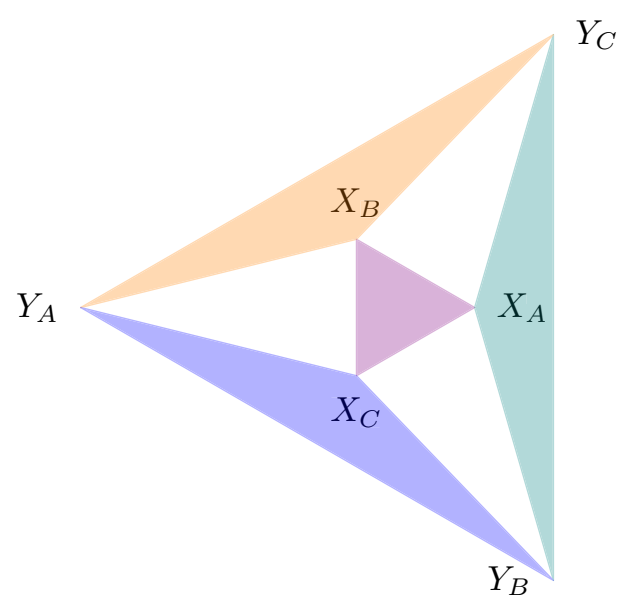

FIG. 6. The abstract simplicial complex forming the base for the Anders-Browne example.

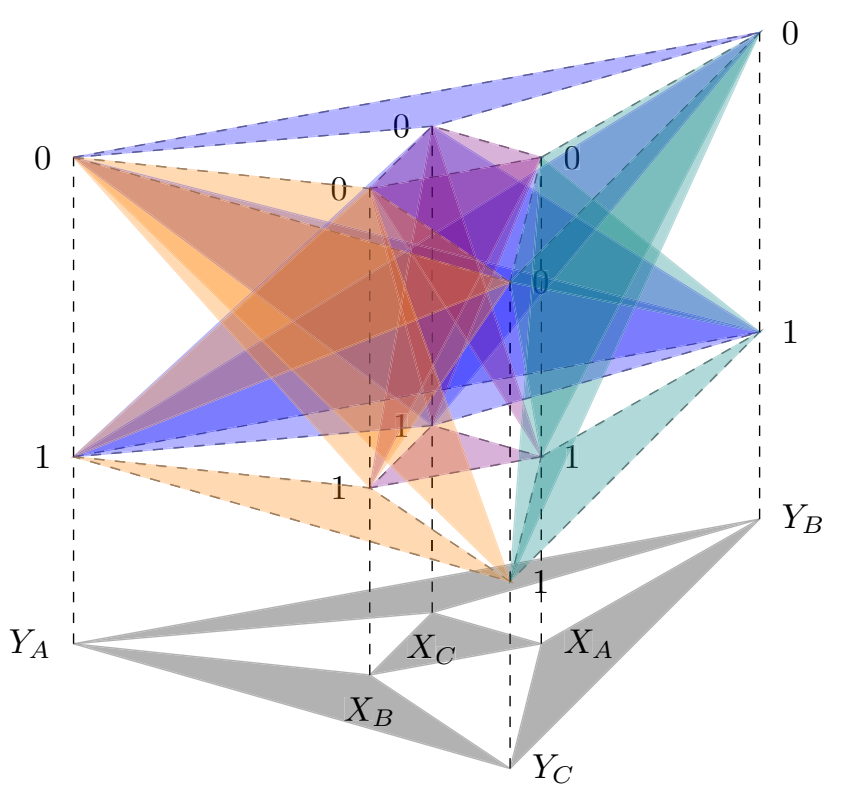

FIG. 7. Bundle diagram with all contexts for the Anders-Browne example.

dimensions we can always draw a surface through three points. To build, in a similar way, a bundle diagram for a contextual model involving four or more agents would require a higher dimensional ambient space. In Fig. 8 the violet triangles represent the sections above the context $C_{1}$. The context $C_{2}$ is marked with teal triangles. The sections related to $C_{3}$ are depicted in orange, the ones belonging to $C_{4}$ in blue. Points in the stalk above a measurement are labeled according to the observable and the outcome-for example, the point above $X_{A}$ corresponding to outcome 1 is denoted $X_{A} 1$.

We now explore the contextuality of this model: if there exists a triangle which cannot be continued to a global section, then the model is contextual. We argue this is the case by starting with the violet triangle $X_{A} 1-X_{B} 1-X_{C} 1$ and exploring all potential compatible ways of extending this section to a global section. We illustrate this procedure via a tree diagram, which graphically enumerates all the possible extensions.

We draw a node (connected to our start triangle) in the tree diagram for the assignment of 1 to $X_{A}$, i.e., the point $X_{A} 1$ in the stalk above $X_{A}$. We then draw leaves connected to nodes labeled by triangles containing $X_{A} 1$. Each such triangle node has leaves corresponding to compatible assignments and so on. Thus our tree is comprised of alternating layers of triangles and assignments. We tag the leaves of a node with a + symbol when they are incompatible. Since in a global section only one outcome per measurement is allowed and our start triangle contains $X_{A} 1, X_{B} 1$, and $X_{C} 1$, we stop when we meet any of $X_{A} 0, X_{B} 0$, or $X_{C} 0$.

Of course we also have to connect the edges $X_{B} 1$ and $X_{C} 1$ of the starting triangle correctly. When we meet them again via this extension process, we mark the nodes with $*$ or $* *$. We can see that there is a possibility to connect the edges $X_{B} 1$ and $X_{C} 1$ of the start triangle and touch all measurements $X_{A}, X_{B}, Y_{A}$, and $Y_{B}$ with ones, unless $Y_{A} 1 \neq$ 


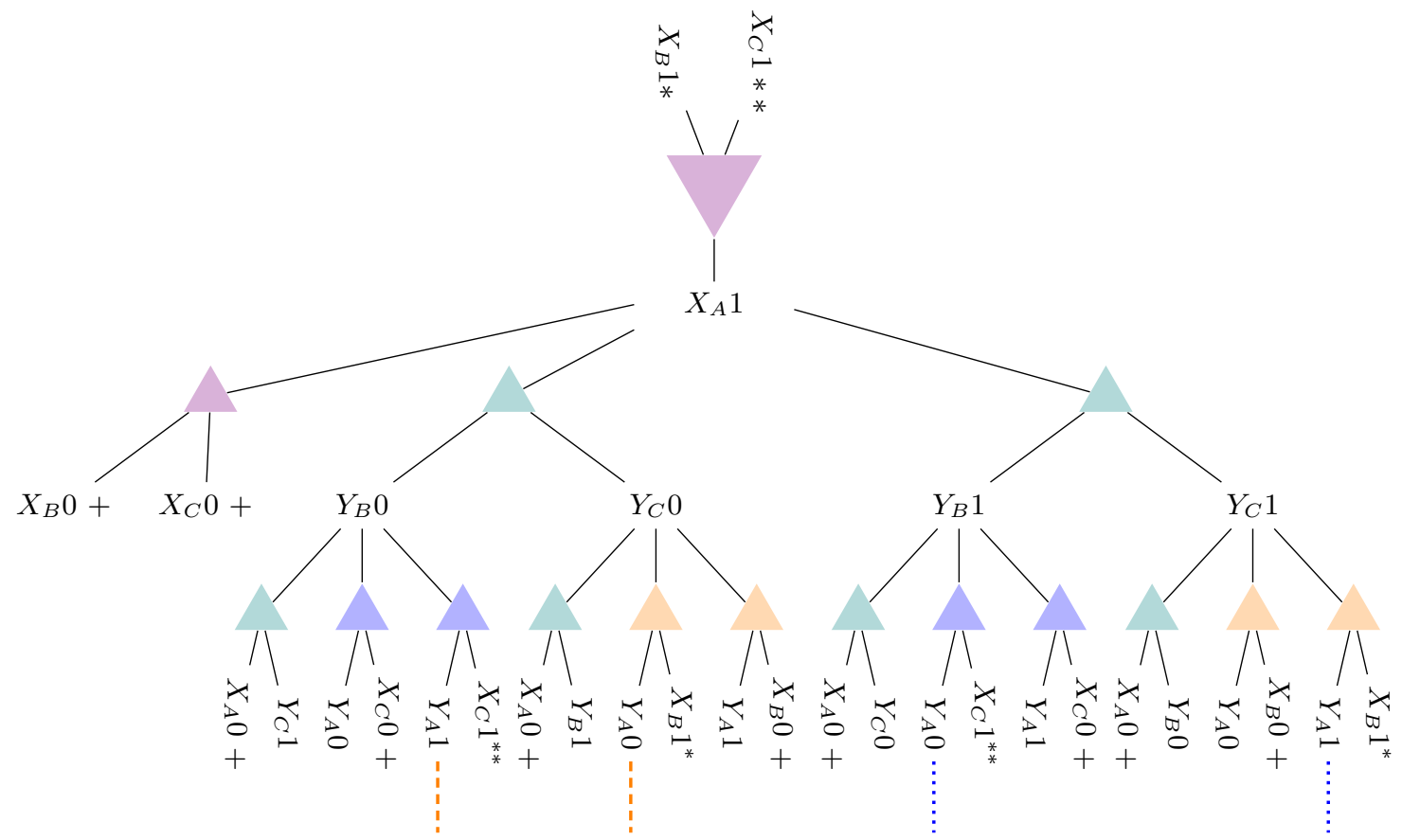

FIG. 8. Trying to build a global section with the triangle $X_{A} 1-X_{B} 1-X_{C} 1$.

$Y_{A} 0$. This situation is tagged with an dashed orange line. A similar case corresponds to the dotted blue lines. We can see graphically how building a global section fails in these two cases in Fig. 9.

Using such a tree diagram, we can also see that it is not possible to build a complete global section with the triangle $X_{A} 1-X_{B} 0-X_{C} 0$.

According to this argument, the model is logically contextual. We can further deduce that the model is strongly contextual, i.e., there is no global section at all. (Because logical contextuality follows from strong contextuality, the first proof is not needed but is mentioned for illustrating techniques before.) To this end, we define a function $f$ : $\operatorname{Support}[P(O)] \rightarrow\{ \pm 1\}$ for each context, where $P(O)$ is the possibility to get the outcome $O=(a, b, c)$ via the joint measurement of the context. Considering $C_{1}$, the function is defined by
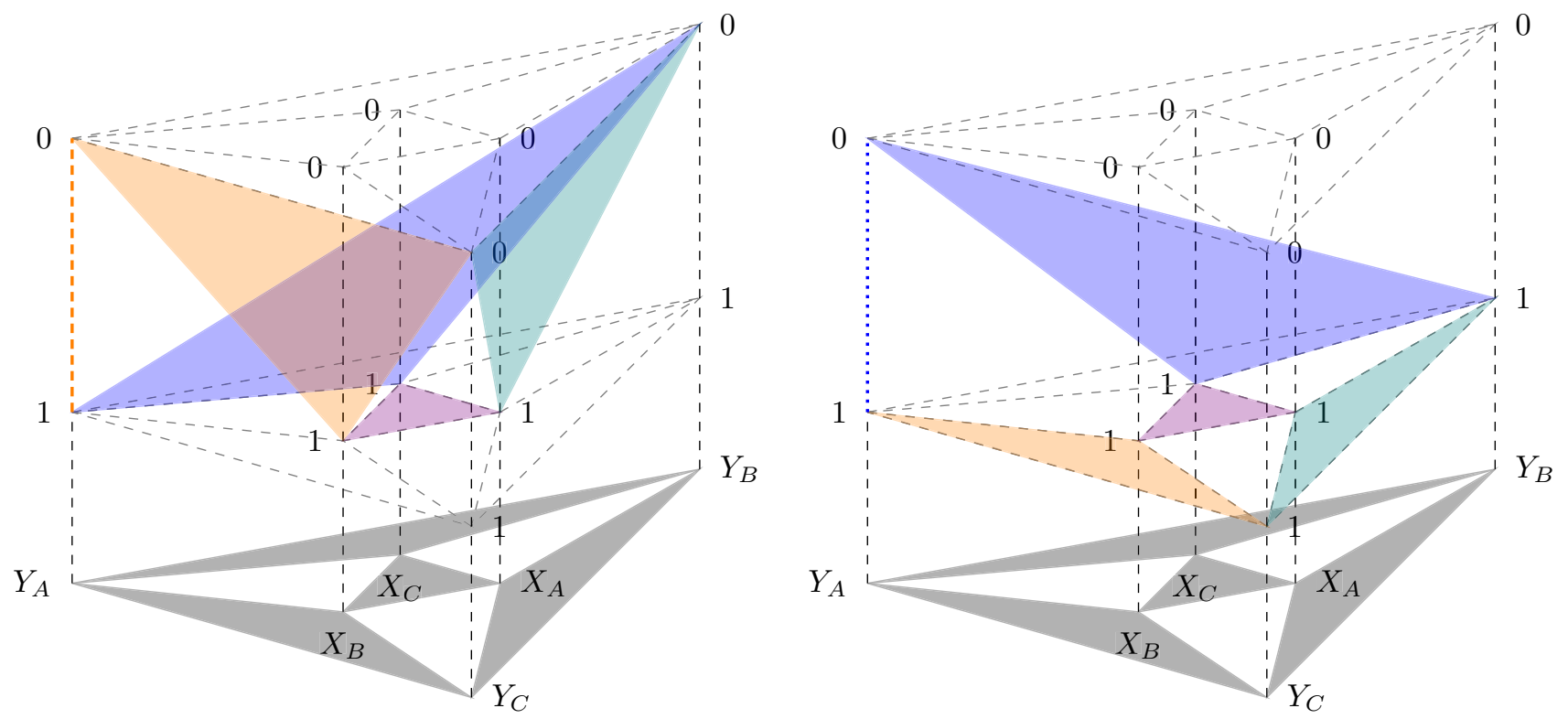

FIG. 9. Examples for failed global sections for the Anders-Browne example. 


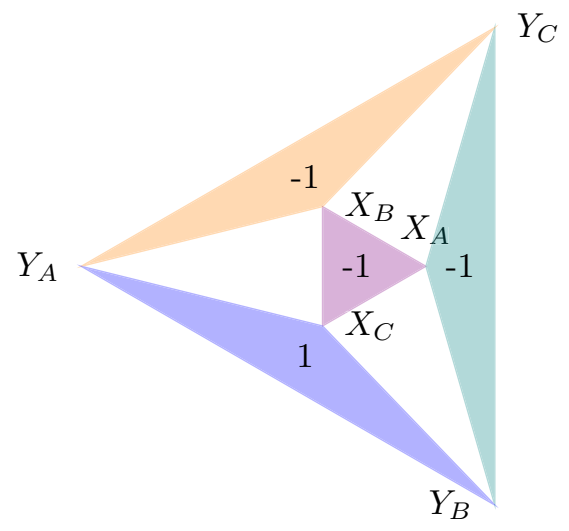

FIG. 10. Values of parity function.

and produces the following codomain:

$$
\begin{aligned}
& f_{C_{1}}(0,0,1)=-1, \\
& f_{C_{1}}(0,1,0)=-1, \\
& f_{C_{1}}(1,0,0)=-1, \\
& f_{C_{1}}(1,1,1)=-1 .
\end{aligned}
$$

Defining the other functions analogously and computing the codomains, we observe that the codomain for one particular context is either $\{-1\}$ or $\{1\}$ so we can summarize the functions $f_{C_{i}}$ for $i \in\{1, \ldots, 4\}$ with an overall parity function $F: C_{i} \rightarrow\{ \pm 1\}$ defined on $\left\{C_{i} \mid i \in\{1, \ldots, 4\}\right\}$. We depict the values of the parity function for each context in Fig. 10.

It follows that all triangles have an odd number of edges on the one-plane, except for the blue ones. So it is not possible to build any global section. Also the structure of the NAND gate becomes clear in this approach, i.e.,

$$
\begin{gathered}
X_{A} X_{B} X_{C}|\Psi\rangle=-|\Psi\rangle, \\
X_{A} Y_{B} Y_{C}|\Psi\rangle=-|\Psi\rangle, \\
Y_{A} X_{B} Y_{C}|\Psi\rangle=-|\Psi\rangle, \\
Y_{A} Y_{B} X_{C}|\Psi\rangle=|\Psi\rangle .
\end{gathered}
$$

We were not able to find a global section using the state $|\Psi\rangle=\frac{1}{\sqrt{2}}(|001\rangle-|110\rangle)$. As a next step, we want to figure out whether there exists a state which produces a model with a global section using the same contexts. Thus we search for a model which fulfills $F\left(C_{i}\right)=-1$ for an even number of contexts $C_{i}$. An appropriate set of conditions on $|\Psi\rangle$ is depicted in Fig. 11 and requires

$$
\begin{gathered}
X_{A} X_{B} X_{C}|\Psi\rangle=-|\Psi\rangle, \\
X_{A} Y_{B} Y_{C}|\Psi\rangle=|\Psi\rangle,
\end{gathered}
$$

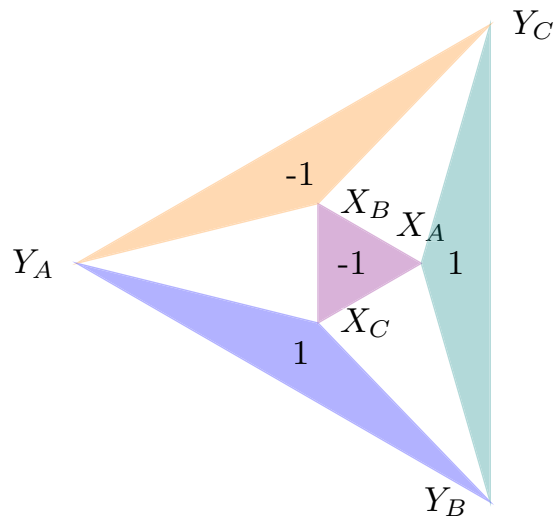

FIG. 11. Values of parity function.

$$
\begin{gathered}
Y_{A} X_{B} Y_{C}|\Psi\rangle=-|\Psi\rangle, \\
Y_{A} Y_{B} X_{C}|\Psi\rangle=|\Psi\rangle .
\end{gathered}
$$

To build such a state $|\Psi\rangle$ fulfilling these conditions, we construct the projectors

$$
\begin{aligned}
P_{1} & =\frac{\mathbb{1}-X_{A} X_{B} X_{C}}{2}, \\
P_{2} & =\frac{\mathbb{1}+X_{A} Y_{B} Y_{C}}{2}, \\
P_{3} & =\frac{\mathbb{1}-Y_{A} Y_{B} X_{C}}{2}, \\
P_{4} & =\frac{\mathbb{1}+X_{A} X_{B} Y_{C}}{2} .
\end{aligned}
$$

If $P_{1}|\Psi\rangle=P_{2}|\Psi\rangle=P_{3}|\Psi\rangle=P_{4}|\Psi\rangle=|\Psi\rangle$ and these projectors come from commuting generators, three of which are independent, then $\operatorname{tr}\left(P_{1} P_{2} P_{3} P_{4}\right)=1$ and therefore $P_{1} P_{2} P_{3} P_{4}=|\Psi\rangle\langle\Psi|[41]$. In the case depicted in Fig. 11, we get $P_{1} P_{2} P_{3} P_{4}=0$. Hence there is no such state $|\Psi\rangle \neq 0$.

Calculating the states resulting from all sets of conditions satisfying $F\left(C_{i}\right)=-1$ for an even number of the four contexts $C_{i}$ analogously, we notice an interesting fact (Lemma 6).

Lemma 6: The states with an even number of minus signs in the projectors vanish using the contexts

$$
\begin{gathered}
C_{1}=\left\{X_{A}, X_{B}, X_{C}\right\}, \\
C_{2}=\left\{X_{A}, Y_{B}, Y_{C}\right\}, \\
C_{3}=\left\{Y_{A}, X_{B}, Y_{C}\right\}, \quad \text { and } \\
C_{4}=\left\{Y_{A}, Y_{B}, X_{C}\right\} .
\end{gathered}
$$

It is only possible to find a state $|\Psi\rangle \neq 0$ for an odd number of minus signs in the projectors.

Proof. We can prove this by calculating $P_{1} P_{2} P_{3} P_{4}=$ $|\Psi\rangle\langle\Psi|$ for all cases.

On one hand, we reasoned that for a state with an odd number of minus signs there is no global face. On the other, 
TABLE V. Empirical model for cluster state $n=5$.

\begin{tabular}{cccccccccccccc}
\hline \hline & 1 & 2 & 3 & 4 & 5 & 000 & 001 & 010 & 011 & 100 & 101 & 110 & 111 \\
\hline$C_{1}$ & $X_{1}$ & $Z_{2}$ & $\mathbb{1}$ & $\mathbb{1}$ & $Z_{5}$ & $1 / 4$ & 0 & 0 & $1 / 4$ & 0 & $1 / 4$ & $1 / 4$ & 0 \\
$C_{2}$ & $Z_{1}$ & $X_{2}$ & $Z_{3}$ & $\mathbb{1}$ & $\mathbb{1}$ & $1 / 4$ & 0 & 0 & $1 / 4$ & 0 & $1 / 4$ & $1 / 4$ & 0 \\
$C_{3}$ & $\mathbb{1}$ & $Z_{2}$ & $X_{3}$ & $Z_{4}$ & $\mathbb{1}$ & $1 / 4$ & 0 & 0 & $1 / 4$ & 0 & $1 / 4$ & $1 / 4$ & 0 \\
$C_{4}$ & $\mathbb{1}$ & $\mathbb{1}$ & $Z_{3}$ & $X_{4}$ & $Z_{5}$ & $1 / 4$ & 0 & 0 & $1 / 4$ & 0 & $1 / 4$ & $1 / 4$ & 0 \\
$C_{5}$ & $Z_{1}$ & $\mathbb{1}$ & $\mathbb{1}$ & $Z_{4}$ & $X_{5}$ & $1 / 4$ & 0 & 0 & $1 / 4$ & 0 & $1 / 4$ & $1 / 4$ & 0 \\
& 1 & 2 & 3 & 4 & 5 & 0000 & 0001 & 0010 & 0011 & 0100 & 0101 & 0110 & 0111 \\
\hline$C_{6}$ & $Z_{1}$ & $X_{2}$ & $\mathbb{1}$ & $X_{4}$ & $Z_{5}$ & $1 / 4$ & 0 & 0 & $1 / 4$ & 0 & $1 / 4$ & $1 / 4$ & 0 \\
$C_{7}$ & $Z_{1}$ & $Z_{2}$ & $X_{3}$ & $\mathbb{1}$ & $X_{5}$ & $1 / 4$ & 0 & 0 & $1 / 4$ & 0 & $1 / 4$ & $1 / 4$ & 0 \\
$C_{8}$ & $X_{1}$ & $Z_{2}$ & $Z_{3}$ & $X_{4}$ & $\mathbb{1}$ & $1 / 4$ & 0 & 0 & $1 / 4$ & 0 & $1 / 4$ & $1 / 4$ & 0 \\
$C_{9}$ & $\mathbb{1}$ & $X_{2}$ & $Z_{3}$ & $Z_{4}$ & $X_{5}$ & $1 / 4$ & 0 & 0 & $1 / 4$ & 0 & $1 / 4$ & $1 / 4$ & 0 \\
$C_{10}$ & $X_{1}$ & $\mathbb{1}$ & $X_{3}$ & $Z_{4}$ & $Z_{5}$ & $1 / 4$ & 0 & 0 & $1 / 4$ & 0 & $1 / 4$ & $1 / 4$ & 0 \\
& & & & & & 1000 & 1001 & 1010 & 1011 & 1100 & 1101 & 1110 & 1111 \\
$C_{6}$ & $Z_{1}$ & $X_{2}$ & $\mathbb{1}$ & $X_{4}$ & $Z_{5}$ & 0 & $1 / 4$ & $1 / 4$ & 0 & $1 / 4$ & 0 & 0 & $1 / 4$ \\
$C_{7}$ & $Z_{1}$ & $Z_{2}$ & $X_{3}$ & $\mathbb{1}$ & $X_{5}$ & 0 & $1 / 4$ & $1 / 4$ & 0 & $1 / 4$ & 0 & 0 & $1 / 4$ \\
$C_{8}$ & $X_{1}$ & $Z_{2}$ & $Z_{3}$ & $X_{4}$ & $\mathbb{1}$ & 0 & $1 / 4$ & $1 / 4$ & 0 & $1 / 4$ & 0 & 0 & $1 / 4$ \\
$C_{9}$ & $\mathbb{1}$ & $X_{2}$ & $Z_{3}$ & $Z_{4}$ & $X_{5}$ & 0 & $1 / 4$ & $1 / 4$ & 0 & $1 / 4$ & 0 & 0 & $1 / 4$ \\
$C_{10}$ & $X_{1}$ & $\mathbb{1}$ & $X_{3}$ & $Z_{4}$ & $Z_{5}$ & 0 & $1 / 4$ & $1 / 4$ & 0 & $1 / 4$ & 0 & 0 & $1 / 4$ \\
\hline \hline
\end{tabular}

we proved that there is no state $|\Psi\rangle \neq 0$ satisfying $F\left(C_{i}\right)=$ -1 for an even number of $C_{i}$. Thus there is no state producing an empirical model with a global section using the contexts defined at the beginning of the section.

\section{THE CLUSTER STATE ON A RING OF FIVE QUBITS}

We consider now a cluster state model on a ring of $n=5$ qubits. This state is stabilized by five stabilizer operators (the first five below). Taking products we generate five additional stabilizers:

$$
\begin{aligned}
& X Z \mathbb{1} Z|\Psi\rangle=|\Psi\rangle, \\
& \mathbb{1} Z X Z \mathbb{1}|\Psi\rangle=|\Psi\rangle, \\
& Z \mathbb{1} Z X|\Psi\rangle=|\Psi\rangle, \\
& Z Z X \mathbb{1} X|\Psi\rangle=|\Psi\rangle, \\
& \mathbb{1} X Z Z X|\Psi\rangle=|\Psi\rangle, \\
& Z X Z \mathbb{1}|\Psi\rangle=|\Psi\rangle, \\
& \mathbb{1} Z X Z|\Psi\rangle=|\Psi\rangle, \\
& Z X \mathbb{1} X Z|\Psi\rangle=|\Psi\rangle, \\
& X Z Z X \mathbb{1}|\Psi\rangle=|\Psi\rangle, \\
& X \mathbb{1} X Z Z|\Psi\rangle=|\Psi\rangle .
\end{aligned}
$$

We use for our contexts the observables contained in each of these ten stabilizers. These are listed in Table V. The entries

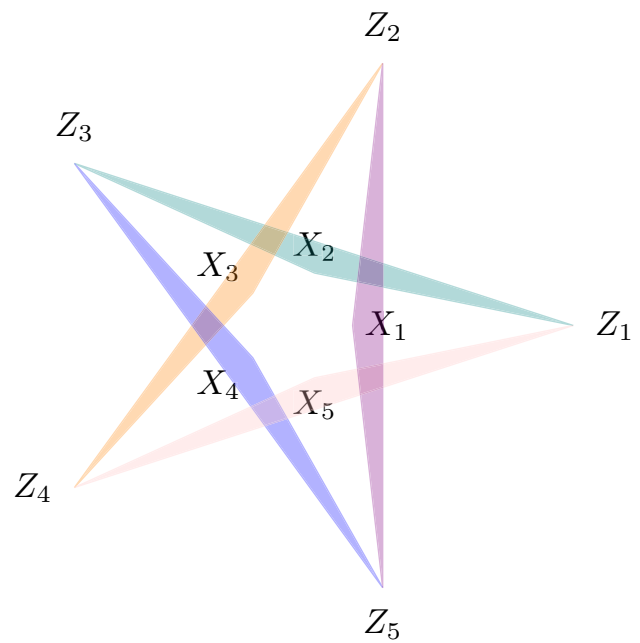

FIG. 12. Base of bundle diagram for the cluster state on a ring of $n=5$ qubits involving contexts $C_{1}-C_{5}$.

with nonzero probabilities are used for building the bundle diagram. For a better overview, we depict the base of the bundle diagram and the bundle diagram itself in two different figures, divided in the contexts $C_{1}$ to $C_{5}$ (base in Fig. 12 and diagram in Fig. 15) and $C_{6}$ to $C_{10}$ (base in Fig. 13 and diagram in Fig. 16). (The complete bundle diagram is the union of these two figures.) Note that since we have contexts involving four observables, we are unable to depict the sections above the corresponding quadrilaterals as flat surfaces; they are buckled in general.

If there exists a triangle or square which cannot be continued to a global section, then the model is contextual. We prove this by considering the teal triangle $Z_{1} 0-X_{2} 0-Z_{3} 0$ and depicting all ways of trying to find a global section via a tree diagram in Fig. 14, as before. We start with the chosen triangle and draw all triangles containing $X_{2} 0$. We can see that only two of the first five children, $A$ and $B$, do not contain a

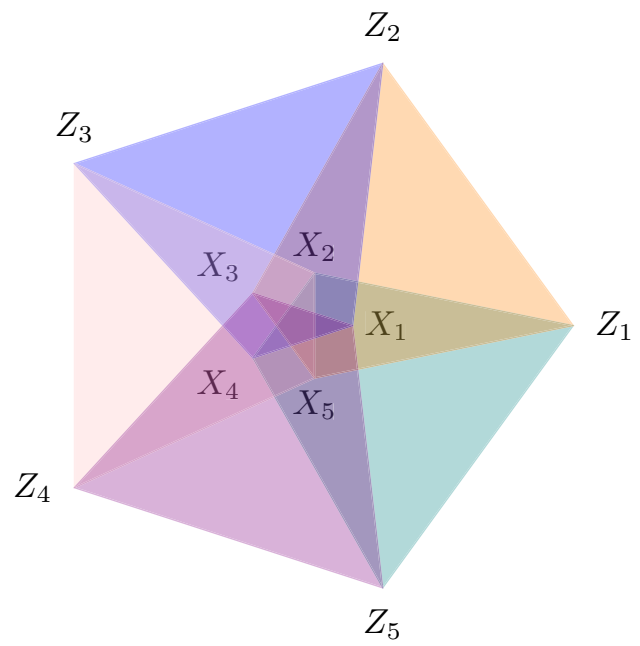

FIG. 13. Base of bundle diagram for the cluster state on a ring of $n=5$ qubits involving contexts $C_{6}-C_{10}$. 


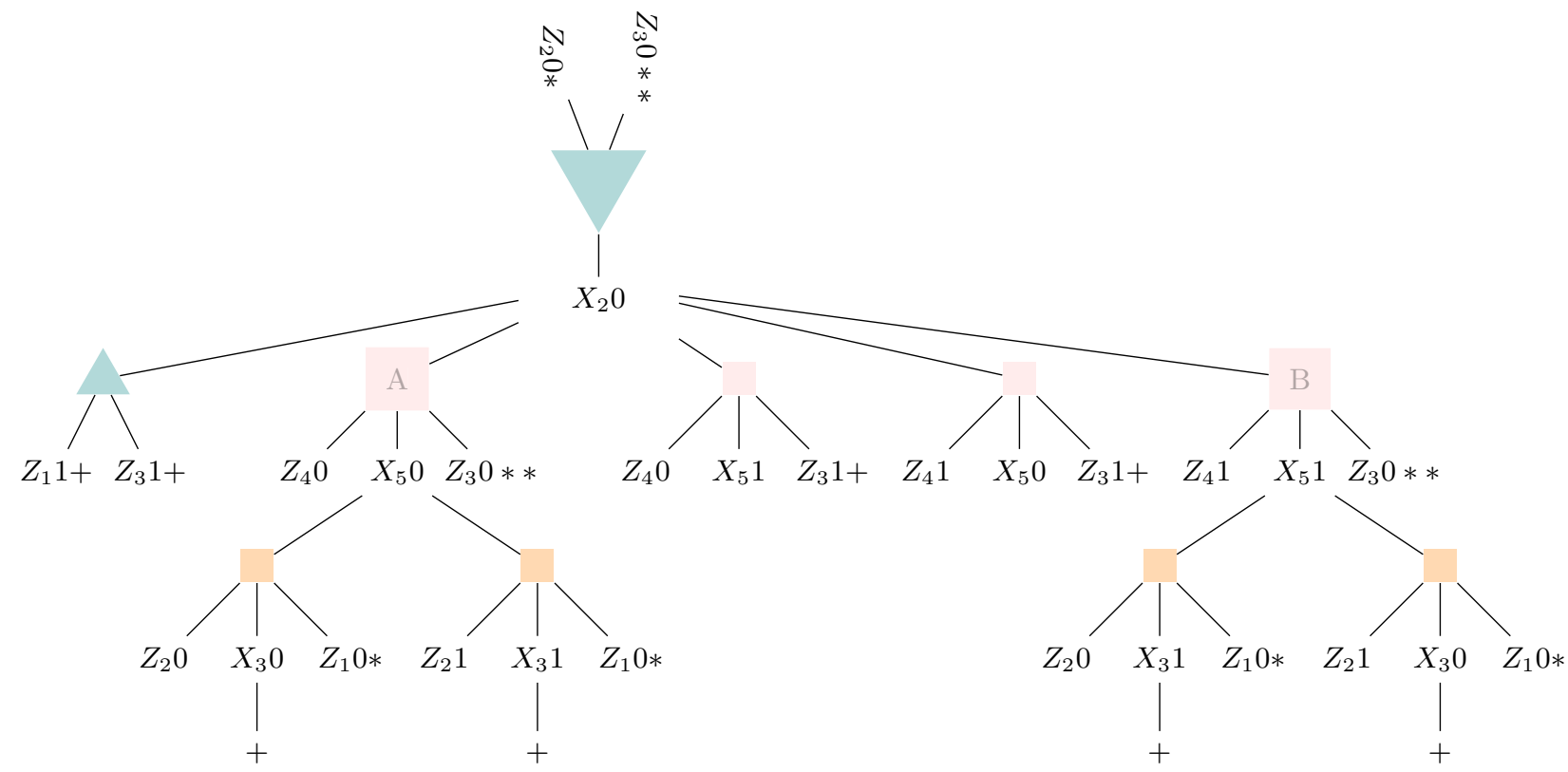

FIG. 14. Trying to build a global section with the triangle $Z_{1} 0-X_{2} 0-Z_{3} 0$.

+ symbol. All vertices of $A$ should be feasible for building a global section with these children. But continuing $X_{5} 0$, one can see that at the end there are no compatible triangles or squares. The case $B$ is similar. We hence cannot build a global section for the triangle $Z_{1} 0-X_{2} 0-Z_{3} 0$ and, therefore, the model is contextual.

\section{CONCLUSION}

The aim of this work was to exploit bundle diagrams to illustrate the contextuality of empirical models involving three or more agents. We considered first bipartite two-agent models with two outcomes as a motivation and then gener-

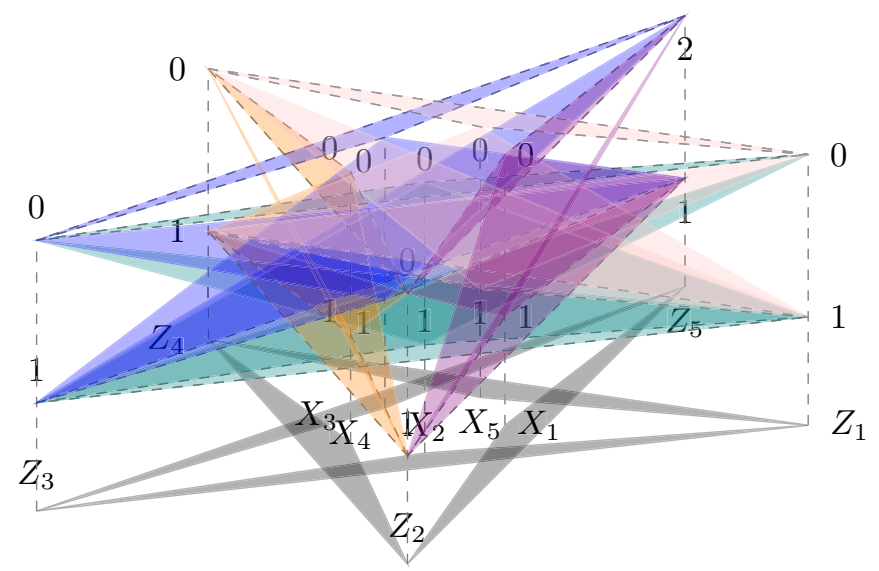

FIG. 15. Bundle diagram for the cluster state on a ring of $n=5$ qubits for contexts $C_{1}-C_{5}$. alized the bundle diagram representation from the two-agent setting to the many-agent setting and illustrated the contextuality of a model built on the Greenberger-Horne-Zeilinger state. This representation was then applied to visualize the contextuality of a joint measurability scenario involving a cluster state for a five-qubit ring. There are many interesting questions that arise in depicting contextuality in this way. For example, the bundle diagram itself is an abstract simplicial complex and the contextuality of the model corresponds to the "twistedness" of the bundle, like for a Möbius strip. Finding a way to quantify the connection between orientability and contextuality more precisely is an intriguing research direction.

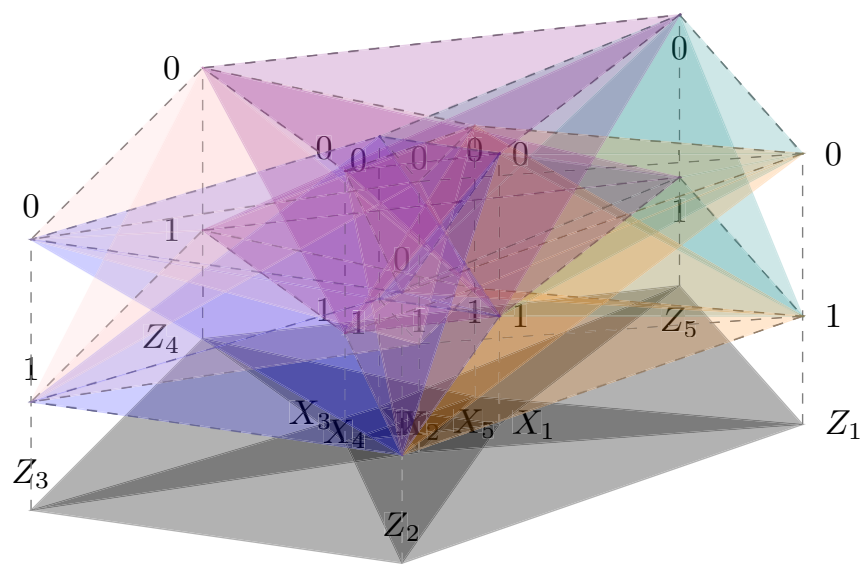

FIG. 16. Bundle diagram for the cluster state on a ring of $n=5$ qubits for contexts $C_{6}-C_{10}$. 


\section{ACKNOWLEDGMENTS}

This work was supported by the the Deutsche Forschungsgemeinschaft through Sonderforschungsbereich 1227
(DQ-mat) and the RTG 1991. Helpful correspondence and discussions with Samson Abramsky, Rui Barbosa, and Dan Browne are gratefully acknowledged.
[1] S. Kochen and E. P. Specker, The Logico-Algebraic Approach to Quantum Mechanics, edited by C. A. Hooker, The University of Western Ontario Series in Philosophy of Science, Vol. 5a (Springer, Netherlands, 1975), pp. 293-328.

[2] J. S. Bell, Physics 1, 195 (1964).

[3] P. Heywood and M. L. G. Redhead, Found. Phys. 13, 481 (1983).

[4] A. Stairs, Philos. Sci. 50, 578 (1983).

[5] N. D. Mermin, Phys. Rev. Lett. 65, 3373 (1990).

[6] R. Raussendorf and T.-C. Wei, Ann. Rev. Cond. Matter Phys. 3, 239 (2012).

[7] R. Raussendorf and H. J. Briegel, arXiv:quant-ph/0010033.

[8] M. A. Nielsen, Rep. Math. Phys. 57, 147 (2006).

[9] D. Gross, J. Eisert, N. Schuch, and D. Perez-Garcia, Phys. Rev. A 76, 052315 (2007).

[10] J. Anders and D. E. Browne, Phys. Rev. Lett. 102, 050502 (2009).

[11] N. Delfosse, P. Allard Guerin, J. Bian, and R. Raussendorf, Phys. Rev. X 5, 021003 (2015).

[12] M. Howard, J. J. Wallman, V. Veitch, and J. Emerson, Nature 510, 351 (2014).

[13] R. Raussendorf, D. E. Browne, N. Delfosse, C. Okay, and J. Bermejo-Vega, Phys. Rev. A 95, 052334 (2017).

[14] A. Cabello, S. Severini, and A. Winter, Phys. Rev. Lett. 112, 040401 (2014)

[15] R. Raussendorf, arXiv:1602.04155.

[16] C. Okay, S. Roberts, S. D. Bartlett, and R. Raussendorf, Quantum Inform. Comput. 17, 1135 (2017).

[17] S. Abramsky, S. Mansfield, and R. S. Barbosa, Electron. Proc. Theor. Comp. Sci. 95, 1 (2012).

[18] S. Abramsky and A. Brandenburger, New J. Phys. 13, 113036 (2011).

[19] E. Amselem, M. Rådmark, M. Bourennane, and A. Cabello, Phys. Rev. Lett. 103, 160405 (2009).

[20] H. Bartosik, J. Klepp, C. Schmitzer, S. Sponar, A. Cabello, H. Rauch, and Y. Hasegawa, Phys. Rev. Lett. 103, 040403 (2009).

[21] A. Cabello, S. Filipp, H. Rauch, and Y. Hasegawa, Phys. Rev. Lett. 100, 130404 (2008).

[22] A. Cabello, Phys. Rev. Lett. 101, 210401 (2008).
[23] G. Kirchmair, F. Zähringer, R. Gerritsma, M. Kleinmann, O. Gühne, A. Cabello, R. Blatt, and C. F. Roos, Nature 460, 494 (2009).

[24] R. Lapkiewicz, P. Li, C. Schaeff, N. K. Langford, S. Ramelow, M. Wieśniak, and A. Zeilinger, Nature 474, 490 (2011).

[25] M. Michler, H. Weinfurter, and M. Żukowski, Phys. Rev. Lett. 84, 5457 (2000).

[26] R. W. Spekkens, D. H. Buzacott, A. J. Keehn, B. Toner, and G. J. Pryde, Phys. Rev. Lett. 102, 010401 (2009).

[27] S. Yu and C. H. Oh, Phys. Rev. Lett. 108, 030402 (2012).

[28] X. Zhang, M. Um, J. Zhang, S. An, Y. Wang, D.-1. Deng, C. Shen, L.-M. Duan, and K. Kim, Phys. Rev. Lett. 110, 070401 (2013).

[29] B. Amaral, M. T. Cunha, and A. Cabello, Phys. Rev. A 92, 062125 (2015)

[30] P. Gnaciński, M. Rosicka, R. Ramanathan, K. Horodecki, M. Horodecki, P. Horodecki, and S. Severini, New J. Phys. 18, 045020 (2016).

[31] P. Kurzyński, Phys. Rev. A 95, 012133 (2017).

[32] R. Raussendorf, D. Wang, A. Prakash, T.-C. Wei, and D. Stephen, Phys. Rev. A 96, 012302 (2017).

[33] D. T. Stephen, D.-S. Wang, A. Prakash, T.-C. Wei, and R. Raussendorf, Phys. Rev. Lett. 119, 010504 (2017).

[34] S. Abramsky, R. S. Barbosa, K. Kishida, R. Lal, and S. Mansfield, Contextuality, Cohomology and Paradox, 24th EACSL Annual Conference on Computer Science Logic (CSL, 2015), Vol. 41, p. 211-228.

[35] G. Carù, Electron. Proc. Theor. Comput. Sci. 236, 21 (2017).

[36] R. Raussendorf, Phys. Rev. A 88, 022322 (2013).

[37] J. Henson, arXiv:1210.5978 .

[38] R. Kunjwal, C. Heunen, and T. Fritz, Phys. Rev. A 89, 052126 (2014).

[39] S. Popescu and D. Rohrlich, Found. Phys. 24, 379 (1994).

[40] D. E. Browne and H. J. Briegel, arXiv:quant-ph/0603226.

[41] M. A. Nielsen and I. L. Chuang, Quantum Computation and Quantum Information (Cambridge University Press, Cambridge, 2000). 